Eidgenössische Anstalt

für das forstliche Versuchswesen CH-8903 Birmensdorf

Institut fédéral

de recherches forestières CH-8903 Birmensdorf

Istituto federale

di ricerche forestali

CH-8903 Birmensdorf

Swiss Federal Institute of Forestry Research CH-8903 Birmensdorf
Januar 1982

Nr. 236

Berichte

Rapports

Rapporti

236

Reports
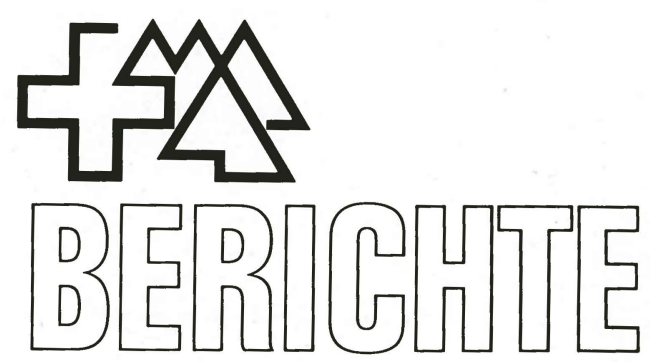

Christian Hoffmann

Die Berechnung von Tarifen

für die Waldinventur

Oxf.: 524.3

Sonderdruck aus Forstwissenschaftliches

Centralblatt 101 (1982) 1: 24 - 36 


\title{
Die Berechnung von Tarifen für die Waldinventur
}

\author{
Von C. Hoffmann
}

\section{Einleitung}

Bei der Waldinventur mit Kontrollstichproben (Schmid-HaAs, Werner, Baumann 1978) werden an einem relativ kleinen Teil der Bäume in den Probeflächen die Durchmesser in $1.3 \mathrm{~m}$ und in $7 \mathrm{~m}$ Höhe und die Scheitelhöhe gemessen. Dieses Verfahren wird laut MaHRER (1981) auch bei der schweizerischen Forstinventur zur Vorratsermittlung angewendet werden. Frühere Untersuchungen haben gezeigt, daß die Aufnahmekosten für eine Vorratsermittlung mit gegebener Genauigkeit für diese Kombination von Meßwerten am geringsten sind und daß die hierzu benötigten dreiparametrigen Volumenfunktionen innerhalb der Schweiz praktisch überall ohne regionale Korrekturen angewendet werden können (Schmid-HaAs 1968). Noch mehr Meßarbeit kann bekanntlich dadurch eingespart werden, daß an vielen Bäumen nur die Durchmesser in $1.3 \mathrm{~m}$ Höhe gemessen werden. Für die Bestimmung der Volumina dieser Bäume sind aber einparametrige Volumenfunktionen oder Tarife

$$
\mathrm{V}=\mathrm{T}(\mathrm{D})
$$

notwendig. Derartige Tarife sind nicht für große Gebiete gültig. Daher müssen lokale Tarife aus denjenigen Volumina berechnet werden, die mit Hilfe der dreiparametrigen Volumenfunktionen aus wenigen Bäumen bestimmt werden.

Die Form der gewählten Tariffunktionen und die Art der Lösung der statistischen, numerischen und programmiertechnischen Probleme bestimmen weitgehend

- die Genauigkeit der Volumenbestimmung durch die Tarife,

- die Anzahl der Bäume pro Tarif, an denen der Durchmesser in $7 \mathrm{~m}$ Höhe und die Scheitelhöhe gemessen werden müssen,

- die Rechenzeit auf dem Rechner und damit die Rechenkosten,

- die Mindestgröße des Rechners, auf dem die Auswertungen noch durchgeführt werden können, insbesondere den Speicherplatzbedarf.

In Kapitel 1 wird die Wahl der Form der Tariffunktion begründet. In den Kapiteln 2 bis 5 werden für den mehr theoretisch orientierten Leser die wichtigsten Eigenschaften des Berechnungsverfahrens dargestellt. Den Praktiker dürften im Kapitel 6 die Eingabedaten, die das Computerprogramm TARIF benötigt, die Wahlmöglichkeiten für die Tarifberechnung und die Form der Ergebnisse interessieren. In Kapitel 7 werden erste Erfahrungen mit dem Programm besprochen.

U.S. Copyright Clearance Center Code Statement: 0015 - 8003/82/10001 - 0024 \$ 02.50/0 Forstw. Cbl. 101 (1982), 24-36 


\section{Die Tariffunktion}

Als Tariffunktionen werden in der Literatur vorwiegend Polynome

$$
T(D)=a_{o}+a_{1} D+a_{2} D^{2}+\ldots+a_{k} D^{k}
$$

erwähnt. Sie sind sehr einfach durch mehrfache lineare Regression berechenbar und leicht $\mathrm{zu}$ handhaben, haben aber die unangenehmen Eigenschaften, Unregelmäßigkeiten in den Daten zu stark zu berücksichtigen und an den Enden des Bereichs von D zu starken Ausschlägen zu neigen, zudem sind negative Volumina nicht auszuschließen.

Funktionen des exponentiellen Typs

$$
T(D)=e^{a_{1}+a_{2} \ln D+\ldots+a_{k} \ln k-1 D}
$$

wurden wegen des hohen Rechenaufwandes bisher kaum verwendet. Sie liefern immer positive Volumina und können zur Glattheit gezwungen werden, indem einige der $a_{i}$ gleich Null gesetzt werden. Langjährige Erfahrungen mit Funktionen dieses Typs (ScHmID-HaAs 1968) motivierten eine Beschränkung der Auswahl auf die drei Funktionen

$$
T(D)=e^{a_{1}+a_{2} \ln D+a_{3} \ln m D}=e^{a_{1}} D^{a_{2}} e^{a_{3} \ln m D}
$$

mit $\mathrm{m}=2$ (PARDE 1961) $\mathrm{m}=4$ (SChmid-HaAs) und $\mathrm{m}=6$. (Ungerade Werte von $\mathrm{m}>2$ wurden nicht berücksichtigt, die grobe Abstufung von m genügte für unsere Zwecke.)

In Abbildung 1 sind für einen Betrieb die Brusthöhendurchmesser D und die Volumina $\mathrm{V}$ derjenigen Probebäume aufgetragen, für die auch der Durchmesser in $7 \mathrm{~m}$ Höhe und die Scheitelhöhe gemessen wurden. Die Kurve der zugehörigen Tariffunktion ist ausgezogen eingezeichnet. Sie paßt gut in den Punkteschwarm hinein. Wir kommen in Kapitel 4 noch auf die Beobachtung zu sprechen, daß die Abweichungen der Volumina von der Tarifkurve mit wachsenden $\mathrm{D}$ größer werden.

Die Interpretation der Eigenschaften der Tariffunktion wird einfacher und die Bedeutung der Parameter wird klarer, wenn wir $\ln \mathrm{D}$ und $\ln \mathrm{T}$ als Variablen betrachten. In Abbildung 2 sind die Daten und die Tarifkurve von Abbildung 1 derart doppelt logarithmisch

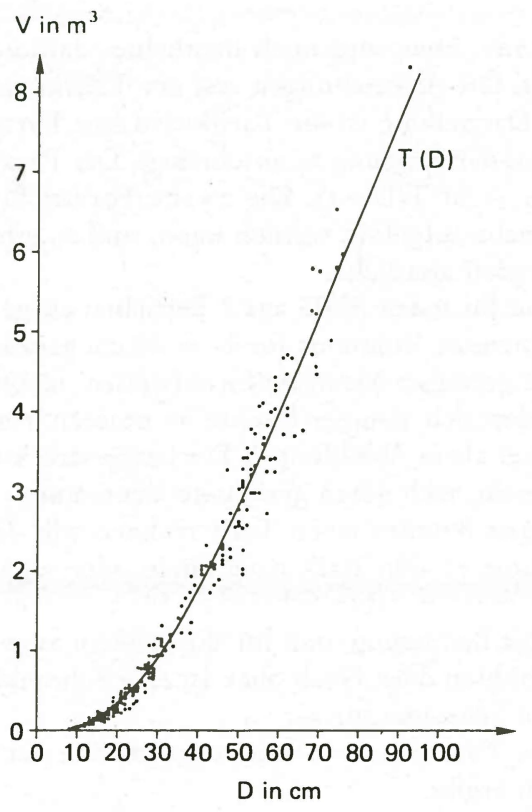

Abb. 1. Brusthöhendurchmesser D und Volumina V von Höhenmessbäumen und zugehörige Tarifkurve

Fig. 1. Diameters D at $1.3 \mathrm{~m}$ and volumes $\mathrm{V}$ of trees whose diameters at $7 \mathrm{~m}$ and heights have also been measured, together with the permanent tarif curve 


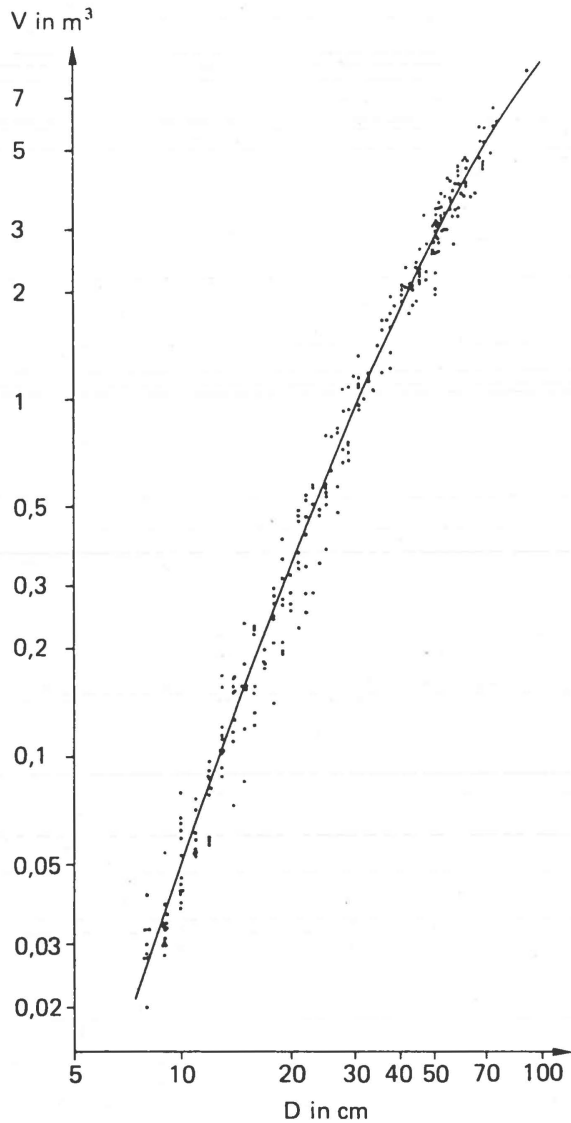

Abb. 2. Daten und Tarifkurven aus Abb. 1 in doppelt-logarithmischer Darstellung

Fig. 2. Data und tarif curves of fig. 1 in doublelogarithmic scale

aufgetragen. Der Punktschwarm ist jetzt fast gerade. Hier wird noch deutlicher, daß die Tarifkurve tatsächlich sehr gut zu den Daten paßt. Die Abweichungen von der Tarifkurve nehmen leicht ab mit zunehmenden D. In dieser Darstellung ist die Tarifkurve eine Parabel, die sich im Punkt ln $\mathrm{D}=0$ an eine Gerade mit der Steigung a anschmiegt. Der Parameter $a_{1}$ beschreibt die Höhenlage des Tarifs: $a_{1}=\ln T(D=1)$. Die zweite Formel für $T(D)$ macht deutlich, daß $a_{2}$ als Wachstumsexponent aufgefaßt werden kann, und $a_{3}$ gibt an, wie stark die Kurve für große D von der Geraden abweicht.

In Abbildung 3 sind die Durchschnittsvolumina für jeden BHD aus 7 Betrieben aufgetragen. Sie sind von links nach rechts nach abnehmenden Volumina für $\mathrm{D}=50 \mathrm{~cm}$ geordnet. Am weitesten links stehen die Betriebe, die die geringste Meereshöhe aufweisen, rechts die mit der größten. Durch die Mittelbildung finden sich weniger Punkte im untèren Teil der Punktewolken, dadurch erscheinen sie schlanker als in Abbildung 3. Die langgestreckte Form fällt sehr deutlich ins Auge, ebenfalls die leicht nach unten gerichtete Krümmung.

Es kann vorkommen, daß man aus sehr wenigen Bäumen einen Tarif rechnen will. In diesem Fall kann die zufällige Lage der Probebäume zu sehr stark nach unten oder sogar nach oben gekrümmten Tarifkurven führen. Wir müssen daher Schranken für $a_{3}$ setzen. Eine brauchbare Schranke nach unten folgt aus der Bedingung, daß für die größten zugelassenen D die Tarifkurve nach rechts hin nicht abfallen darf. Nach oben ist eine Schranke recht willkürlich, wir wählen daher Null als obere Schranke für $a_{3}$.

Anhand von Beispielen wurde ermittelt, daß die Variante $m=4$ meist gerade den richtigen Krümmungsverlauf im $\ln \mathrm{D}$-ln T-Diagramm ergibt. 


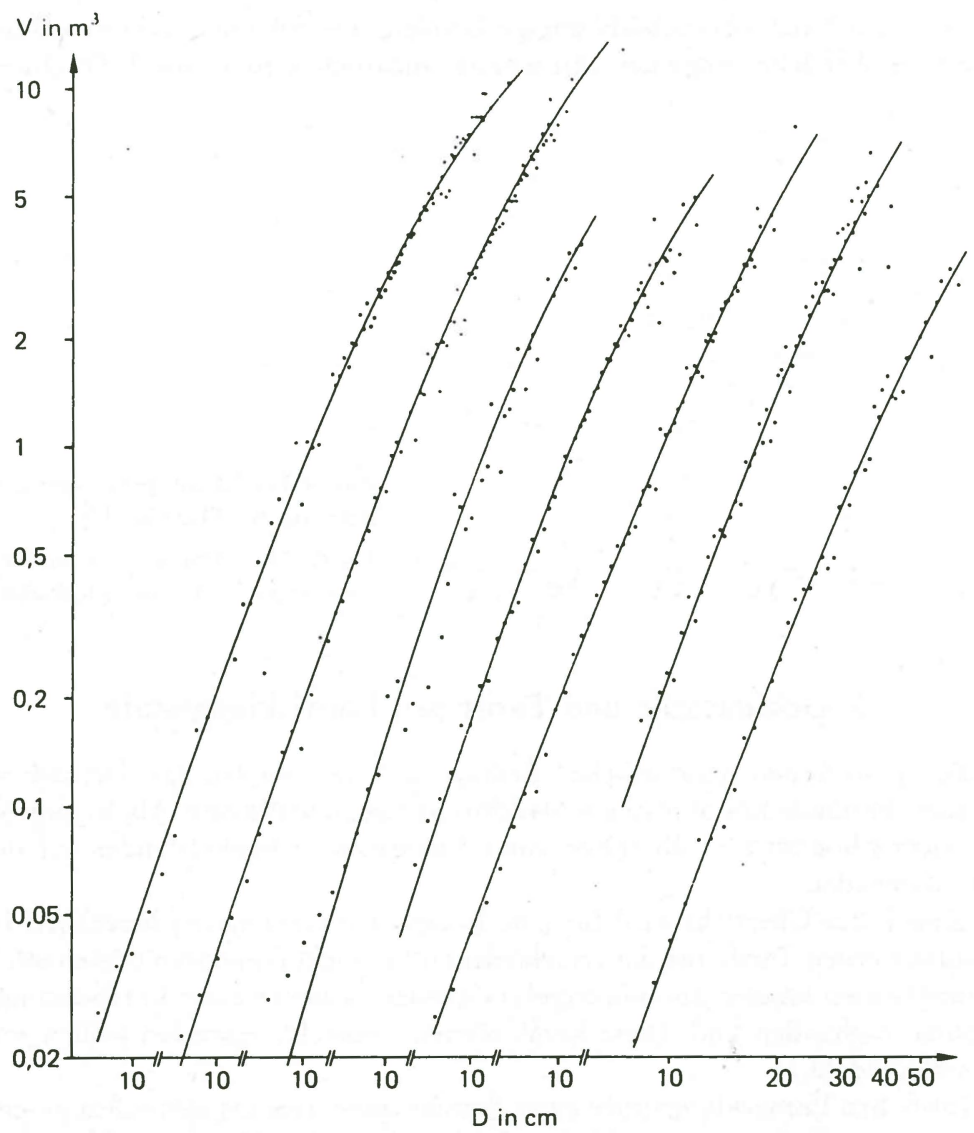

Abb. 3. Durchschnittsvolumina pro BHD und Tarifkurven verschiedener Betriebe in doppelt-logarithmischer Darstellung

Fig. 3. Average volumes per $\mathrm{dbh}$ and tarif curves of various enterprises in double-logarithmic scale

Im weiteren wird also von der Tariffunktion

$$
T(D)=e^{a_{1}+a_{2} \ln D+a_{3} \ln { }^{4} D}=e^{a_{1}} D^{a_{2}} e^{a_{3} \ln ^{4} D}
$$

die Rede sein.

\section{Reduktion der Parameterzahl}

Berechnet man die Tariffunktionen T(D) für die Betriebe, aus denen die Beispiele in Figur 3 stammen, und trägt die so erhaltenen Parameter $a_{1}$ und $a_{2}$ in einem Diagramm auf (s. Abb. 4), so fällt auf, daß die Punkte in einer langgestreckten Wolke angeordnet sind, d.h. $a_{1}$ und $a_{2}$ sind stark miteinander korreliert. Diese Korrelation unter den Parametern kann dazu benutzt werden, einen Parameter aus der Funktion $T$ zu eliminieren. Das hat den Vorteil, daß die Schätzgenauigkeit für die übrigbleibenden Parameter steigt bzw. daß man für eine vorgegebene Genauigkeit an weniger Bäumen den Durchmesser in $7 \mathrm{~m}$ Höhe und die Scheitelhöhe messen muß. Für die bei unseren Rechnungen verwendeten Betriebe ergab sich eine gute Regression

$$
a_{1}=\beta a_{2}+\gamma a_{3}+\delta
$$


wobei in $\beta, \gamma$ und $\delta$ noch betriebsabhängige Größen, wie das Durchschnittsvolumen oder die mittlere Grundfläche, eingehen. Mit diesem Ausdruck wird $a_{1}$ aus T(D) eliminiert.

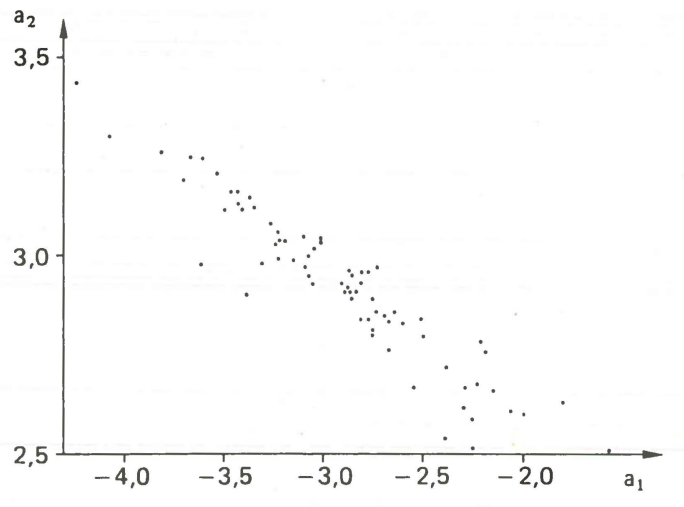

Abb. 4. Die Parameter $\mathrm{a}_{1}$ und $\mathrm{a}_{2}$ für einige Betriebe des Mittellandes

Fig. 4. The parameters $a_{1}$ and $a_{2}$ of some enterprises in the Swiss lowlands

\section{Gesamttarife und Tarife pro Entwicklungsstufe}

Tarife fallen je nach den standörtlichen Bedingungen verschieden aus. Deshalb wird man bestrebt sein, Bestände mit ähnlichem Standort zusammenzufassen. Als Kenngröße dafür wird die Fichtenbonität (= Oberhöhe eines 50jährigen Fichtenbestandes auf demselben Standort) verwendet.

Zum Zweck der Übersicht wird für jede Bonität ein Gesamttarif berechnet. Innerhalb jeder Bonität werden Tarife für die verschiedenen Entwicklungsstufen berechnet. Mehrere Entwicklungsstufen können zusammengefaßt werden, wenn in einer Entwicklungsstufe zu wenig Bäume vorhanden sind. Diese kombinierten Entwicklungsstufen wollen wir wie die einfachen behandeln.

Alle Tarife pro Entwicklungsstufe einer Bonität sollen (bei einigermaßen geschlossenen Beständen) für große D zusammenlaufen, da die sehr dicken Bäume unabhängig vom Bestandestyp eine ähnliche vorherrschende Stellung haben. Das Volumen dieser sehr dicken Bäume wird am zuverlässigsten durch den Gesamttarif wiedergegeben. Da nun für die Tarife pro Entwicklungsstufe meist nur wenige mittlere und sehr dicke Bäume zur Verfügung stehen, erweist es sich als vorteilhaft, zur Stabilisierung einen fiktiven Baum mit dem größten zu verwendenden Durchmesser D (bei uns $127 \mathrm{~cm}$ ) und dessen aus dem Gesamttarif berechneten Volumen in jeden Tarif pro Entwicklungsstufe einzuführen.

\section{Die Gewichtung}

Laut Schmid-HaAs, Werner, Baumann (1978) werden Bäume mit kleinen Brusthöhendurchmessern mit kleinerer Wahrscheinlichkeit erfaßt als solche mit großen. Das ist absichtlich so, weil sehr viel mehr dünne Bäume vorhanden sind und dazu noch die Varianz des Volumens kleiner ist als bei den dickeren. Es werden die Bäume mit einem Durchmesser unter einer bestimmten Grenze nur auf einem ganz genau vorgeschriebenen Teil der Probefläche erfaßt. Sie müssen somit stärker in der Rechnung berücksichtigt werden. Als Gewichtung ist deshalb der Kehrwert der Aufnahmefläche zu nehmen, vgl. CochraN (1972).

Wie wir gesehen haben, streuen die Werte für das Volumen für verschiedene Durchmesser verschieden stark. Will man das durchschnittliche Volumen aller Bäume möglichst genau schätzen, so nimmt man als zusätzliches Gewicht den Reziprokwert der Varianzen. 
Damit werden stärker variierende Werte weniger berücksichtigt. Die Varianz steigt mit $D$, und zwar zwischen quadratisch und biquadratisch. Das ergibt Gewichte zwischen D-2 und $D^{-4}$. Wir wollen die Gewichtung eher nicht zu stark werden lassen und wählen daher D-2.

Die zusätzliche Gewichtung und die Nichtlinearität der Funktion T bezüglich der Parameter bewirken eine Verfälschung der Schätzung des Durchschnittsvolumens. Auf dessen Beseitigung wird am Ende des Abschnitts 5.1 und in HoffmanN (1982b) näher eingegangen.

\section{Die Bestimmung der Parameter}

Die folgenden Ausführungen geben eine abgekürzte Beschreibung des Lösungsverfahrens, das in HofFMANN (1982b) ausführlicher hergeleitet wird.

\subsection{Grundgleichungen}

Die Parameter werden nach der Methode der Kleinsten Quadrate bestimmt, d.h. es wird die gewichtete Varianz $S$

zum Minimum gemacht. Dabei bedeuten

$$
S\left(a_{2}, a_{3}\right)=\sum_{i} G_{i}\left(V_{i}-T_{i}\right)^{2}
$$

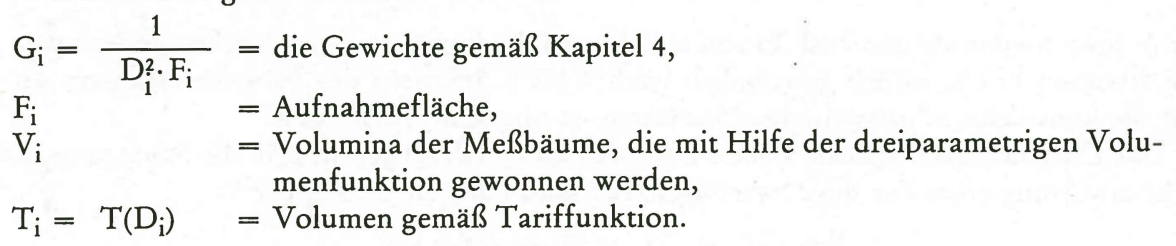

Notwendige Bedingungen für das Minimum von $S$ sind

$$
\begin{aligned}
& \frac{\partial S}{\partial a_{2}}=2 \sum_{i} G_{i} T_{i}\left(V_{i}-T_{i}\right) \cdot\left(\beta+\ln D_{i}\right)=0, \\
& \frac{\partial S}{\partial a_{3}}=2 \sum_{i} G_{i} T_{i}\left(V_{i}-T_{i}\right) \cdot\left(\gamma+\ln ^{4} D_{i}\right)=0 .
\end{aligned}
$$

Für diese in $a_{2}$ und $a_{3}$ nichtlinearen Gleichungen können keine exakten Lösungen angegeben werden. Man muß also Startwerte $a_{2}{ }^{(0)}$ und $a_{3}{ }^{(0)}$ suchen, die die Gleichungen näherungsweise erfüllen und diese dann mit einem Iterationsverfahren verbessern.

Wir approximieren $T_{i}$ durch $V_{i}$. Für $V_{i}-T_{i}$ erhalten wir folgendermaßen eine Näherung: Es gilt

$$
\frac{\ln \mathrm{V}-\ln \mathrm{T}}{\mathrm{V}-\mathrm{T}} \doteq \frac{\lim }{\mathrm{V} \rightarrow \mathrm{T}} \frac{\ln \mathrm{V}-\ln \mathrm{T}}{\mathrm{V}-\mathrm{T}}=\frac{\mathrm{d}(\ln \mathrm{T})}{\mathrm{dT}}=\frac{1}{\mathrm{~T}} \doteq \frac{1}{\mathrm{~V}}
$$

( $\doteq$ bedeutet ungefähr gleich).

Die Näherung für $V_{i}-T_{i}$ ist also $\left(\ln V_{i}-\ln T_{i}\right) V_{i}$. Setzen wir diese Näherungen in die obigen Gleichungen ein, so erhalten wir linerare Gleichungen für $\mathrm{a}_{2}{ }^{(0)}$ und $\mathrm{a}_{3}(0)$ :

$$
\begin{aligned}
& \sum_{\mathrm{i}} \mathrm{G}_{\mathrm{i}} \mathrm{V}_{\mathrm{i}}^{2}\left(\beta+\ln \mathrm{D}_{\mathrm{i}}\right)^{2} \cdot \mathrm{a}_{2}(0)+\sum_{\mathrm{i}} \mathrm{G}_{\mathrm{i}} \mathrm{V}_{\mathrm{i}}^{2}\left(\beta+\ln \mathrm{D}_{\mathrm{i}}\right)\left(\gamma+\ln { }^{4} \mathrm{D}_{\mathrm{i}}\right) \cdot \mathrm{a}_{3}(0) \\
&+\sum_{\mathrm{i}} \mathrm{G}_{\mathrm{i}} \mathrm{V}_{\mathrm{i}}^{2}\left(\beta+\ln \mathrm{D}_{\mathrm{i}}\right)\left(\delta-\ln \mathrm{V}_{\mathrm{i}}\right)=0 \\
& \sum_{\mathrm{i}} \mathrm{G}_{\mathrm{i}} \mathrm{V}_{\mathrm{i}}{ }^{2}\left(\beta+\ln \mathrm{D}_{\mathrm{i}}\right)\left(\gamma+\ln ^{4} \mathrm{D}_{\mathrm{i}}\right) \mathrm{a}_{2}(0)+\sum_{\mathrm{i}} \mathrm{G}_{\mathrm{i}} \mathrm{V}_{\mathrm{i}}^{2}\left(\gamma+\ln \mathrm{D}_{\mathrm{i}}\right)^{2} \cdot \mathrm{a}_{3}(0) \\
&+\sum_{\mathrm{i}} \mathrm{G}_{\mathrm{i}} \mathrm{V}_{\mathrm{i}}^{2}\left(\gamma+\ln \mathrm{D}_{\mathrm{i}}\right)\left(\delta-\ln \mathrm{V}_{\mathrm{i}}\right)=0 .
\end{aligned}
$$

Zur Verbesserung dieser Näherungslösung benutzen wir das Iterationsverfahren von Newton: Die Verbesserungen $\Delta \mathrm{a}_{2}$ und $\Delta \mathrm{a}_{3}$ für die Werte $\mathrm{a}_{2}(\mathrm{j})$ und $\mathrm{a}_{3}(\mathrm{j})$ gewinnen wir durch Linearisierung der partiellen Ableitungen von $S$ im Punkte $\left(a_{2}(j), a_{3}(j)\right)$ : 


$$
\begin{aligned}
& \frac{\partial S}{\partial a_{2}} \doteq \frac{\partial S}{\partial a_{2}}+\frac{\partial^{2} S}{\left(\partial a_{2}\right)^{2}} \Delta a_{2}+\frac{\partial^{2} S}{\partial a_{2} \partial a_{3}} \Delta a_{3}, \\
& \frac{\partial S}{\partial a_{3}} \doteq \frac{\partial S}{\partial a_{3}}+\frac{\partial^{2} S}{\partial a_{2} \partial a_{3}} \Delta a_{2}+\frac{\partial^{2} S}{\left(\partial a_{3}\right)^{2}} \Delta a_{3},
\end{aligned}
$$

wobei die linke Seite für $a_{2}(j+1)$ und $a_{3}(j+1)$ und die rechte Seite für $a_{2}(j)$ und $a_{3}(j) z u$ nehmen sind. Die neuen Parameterwerte sind dann

$$
a_{2}(j+1)=a_{2}(j)+\Delta a_{2}, \quad a_{3}(j+1)=a_{3}(j)+\Delta a_{3} .
$$

Die Berechnung der $a_{2}(j)$ und $a_{3}(j)$ wird für $j=1,2,3, \ldots$ solange fortgesetzt, bis die relative Abnahme von $\mathrm{S}$ unter eine vorgegebene Schranke gesunken ist, also z.B.:

$$
\frac{\left|S\left(a_{2}{ }^{(n-1)}, a_{3}(n-1)\right)-S\left(a_{2}{ }^{(n)}, a_{3}{ }^{(n)}\right)\right|}{S\left(a_{2}{ }^{(n)}, a_{3}{ }^{(n)}\right)}<10^{-5} .
$$

Die Werte $a_{2}=a_{2}(n), a_{3}=a_{3}(n)$ und $a_{1}=a_{1}(n)=\beta a_{2}(n)+\gamma a_{3}(n)+\delta$ betrachten wir dann als Lösung des Minimalproblems für S. Damit ist die Funktion T(D) bestimmt.

Es kann nun vorkommen, daß in einem Iterationsschritt der Wert von S nicht ab- sondern zunimmt. Dann werden die Schrittweiten solange fortlaufend verkleinert

$$
\left(\Delta \mathrm{a}_{2}, \Delta \mathrm{a}_{3}=\frac{1}{4} \Delta \mathrm{a}_{2}, \frac{1}{4} \Delta \mathrm{a}_{3} ; \quad \frac{1}{4} \cdot \frac{1}{8} \Delta \mathrm{a}_{2}, \frac{1}{4} \cdot \frac{1}{8} \Delta \mathrm{a}_{3} ; \quad \frac{1}{4} \cdot \frac{1}{8} \cdot \frac{1}{16} \Delta \mathrm{a}_{2}, \frac{1}{4} \cdot \frac{1}{8} \cdot \frac{1}{16} \Delta \mathrm{a}_{3},\right.
$$

usw.), bis S wieder kleiner wird. In jedem Fall wird die Iteration nach 15 Schritten beendet. Die Iteration bricht jedoch gewöhnlich nach 2 bis 3 Schritten des Newtonverfahrens ab und die künstliche Schrittweitenverkleinerung ist nur sehr selten nötig.

Das Durchschnittsvolumen $\bar{V}$ wird nur ungefähr wiedergegeben, d.h. die Schätzung ist nicht erwartungstreu. Für die Erwartungstreue muß daher nach Kapitel 4

$$
\sum_{\mathrm{i}} \mathrm{F}_{\mathrm{i}}-1 \mathrm{~T}_{\mathrm{i}}=\sum_{\mathrm{i}} \mathrm{F}_{\mathrm{i}}-1 \mathrm{~V}_{\mathrm{i}}=\sum_{\mathrm{i}} \mathrm{F}_{\mathrm{i}}-1 \cdot \overline{\mathrm{V}}
$$

gelten. Die Minimalgleichungen am Anfang des Kapitels können als

$$
\sum\left[D_{i}^{-2}(\ldots)\right] F_{i}-1 T_{i}=\sum[] F_{i}-1 V_{i}
$$

gelesen werden. Der Faktor in der eckigen Klammer ist also für die Verfälschung verantwortlich. Er rührt von der zusätzlichen Gewichtung $\mathrm{D}^{-2}$ und von der Nichtlinearität der Funktion $T$ in den $a_{j}$ her. Am Schluß der Iteration wird daher $a_{1}$ gerade so verändert, daß die Erwartungstreue wiederhergestellt wird. Diese Anpassung mit $a_{1}$ ist wesentlich durchsichtiger und weniger aufwendig als mit $a_{2}$ oder $a_{3}$. Sie vergrößert in vielen Fällen die Summe $S$ wieder etwas, es kann aber auch vorkommen, daß sie durch diese Maßnahme noch ein wenig abnimmt, denn jetzt wird die Bedingung für den Parameter $a_{1}$ außer Kraft gesetzt, die wir im Kapitel 2 besprochen haben. Diese Zwangsmaßnahme nehmen wir in Kauf, denn das Rechenverfahren neigt zur Instabilität beim Versuch, analog dem in Kapitel 5.2 beschriebenen Vorgehen die Erwartungstreue bei jedem Schritt einzuhalten. Das kommt daher, daß im wesentlichen nur noch ein Parameter bestimmbar bleibt.

\subsection{Bedingungen für die Parameter}

Wie wir in Kapitel 1 gesehen haben, soll $a_{3}$ zwei Bedingungen erfüllen. Die eine lautet $a_{3} \leq 0$ und die andere $d T / d D=T \cdot\left(4 a_{3} \ln ^{3} D+a_{2}\right) / D=0$ für $D \leq 130 \mathrm{~cm}$. Es genügt, die zweite Bedingung für $\mathrm{D}=130 \mathrm{~cm}$ zu erzwingen, dann ist sie auch für $\mathrm{D}<130 \mathrm{~cm}$ erfüllt.

Ist nun eine der beiden Bedingungen verletzt, so wird die Einhaltung durch die Lösung des abgeänderten Problems

$$
\mathrm{S}_{\mathrm{B}}=\mathrm{S}+\lambda \cdot \mathrm{a}_{3}=\text { stationär } \quad \text { bzw. }
$$




$$
\mathrm{S}_{\mathrm{B}}=\mathrm{S}+\lambda \cdot\left(\mathrm{a}_{2}+4 \ln ^{3} 130 \cdot \mathrm{a}_{3}\right)=\text { stationär }
$$

erreicht. Das Gleichungssystem

$$
\frac{\partial S_{B}}{\partial a_{2}}=0, \frac{\partial S_{B}}{\partial a_{3}}=0, \frac{\partial S_{B}}{\partial \lambda}=0
$$

bestimmt $a_{2}$ und $a_{3}$ und den nicht weiter interessierenden Lagrangemultiplikator $\lambda$.

\section{Die Struktur des Computerprogramms}

Das TARIF genannte Computerprogramm benötigt zwei Sätze von Eingangsdaten, um daraus die Tarife zu berechnen und entsprechende Ausgangsdaten zu liefern, genaue Angaben finden sich in Hoffmann 1982a und Vogel 1981. Die große Menge der Eingangsdaten ist in der Inventurdatei gespeichert. Daneben werden einige wenige Kontrolldaten auf den Leitkarten verlangt. Ausgegeben werden die Kontrolliste, sogenannte Tarifkarten und eine Inventurdatei mit Einzelbaumvolumina (s. Abb. 5).

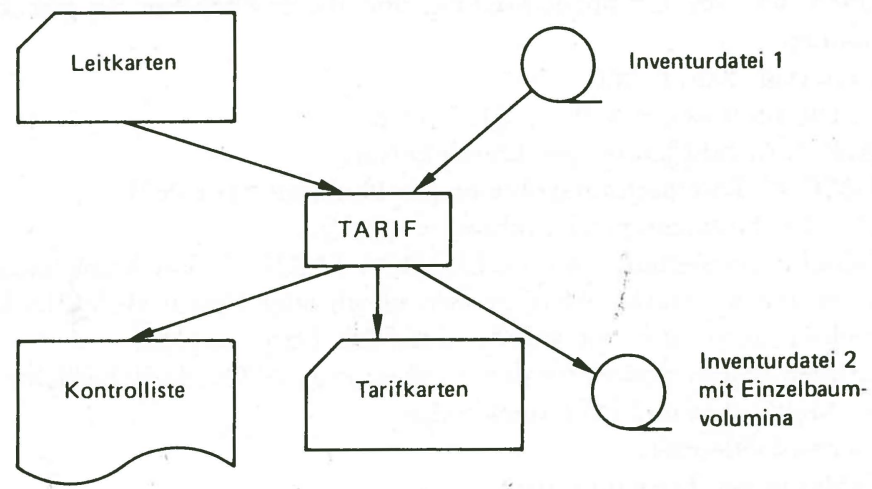

Abb. 5. Grobdatenflußplan des Programms TARIF

Fig. 5. Flow chart of input and output data of the computer programme TARIF

\subsection{Die Eingabedaten}

Die Inventurdatei 1 enthält die bereinigten Einzelbaumdaten der Inventur gemäß VoGEL (1981), nach Probeflächen und Auswerteeinheiten geordnet. Die uns interessierenden Größen sind

$$
\begin{aligned}
& \text { Baumart, } \\
& \text { D } \quad[\mathrm{cm}] \text { der neuen Aufnahme } \\
& \text { D7 }[\mathrm{cm}] \text { der neuen Aufnahme soweit vorhanden } \\
& \text { Höhe [m] der neuen Aufnahme }
\end{aligned}
$$

Die Leitkarten enthalten Parameter und Texte zur Steuerung des Programmablaufs. Mit ihnen wird bestimmt:

1. Welche Probeflächengrößen sind je nach dem Brusthöhendurchmesser D für die Aufnahmewahrscheinlichkeit und damit für die Gewichtung maßgebend, z.B. Bäume mit D $<20 \mathrm{~cm}$ sind auf $1 / 16$ a, mit $\mathrm{D}<50 \mathrm{~cm}$ auf $1 / 4$ a und solche mit $\mathrm{D} \geq 50 \mathrm{~cm}$ auf 3 a großen Probeflächen aufgenommen worden.

2. Alle Waldteile mit gleicher Bonität werden zu einer Gruppe zusammengefaßt. Für jede Gruppe wird zusätzlich ein Gesamttarif berechnet.

3. Welche Waldteile sollen zu einer Entwicklungsstufe zusammengefaßt werden. 
4. Für jede Gruppe kann verlangt werden, daß

a. gemeinsame Tarife für Laub- und für Nadelholz berechnet werden,

b. Laub- und Nadelholz getrennte Tarife bekommen, und zwar in 2 Varianten:

b1. die Mindestanzahl an Bäumen ist sehr klein (z.B. 6 für einen Entwicklungstarif) und die vorkommenden $\mathrm{D}$ müssen nicht sehr stark verschieden voneinander sein (z. B. kleinster D = $10 \mathrm{~cm}$, größter D = $18 \mathrm{~cm}$ ); Gefahr: schlechte Tarife;

b2. die Mindestzahl ist größer (z.B. 12), und die Spanne der D muß größer sein (z. B. größter minus kleinster D mindestens $20 \mathrm{~cm}$ ).

Werden diese Mindestanforderungen unterschritten, so ist ein schlecht angepaßter oder wenig aussagekräftiger Tarif zu erwarten. Er wird daher nicht berechnet. Seine Bäume werden dem Entwicklungstarif der anderen Holzart zugewiesen und erscheinen auch in jenem Gesamttarif.

\subsection{Die Ausgabedaten}

Die Kontrolliste enthält:

1. einen Kontrollausdruck der Leitkarten;

2. Informationen über die Gruppeneinteilung und die gewünschte Art der Laub-Nadelholzbehandlung;

3. die Tariflisten (vgl. Tab. 1) mit

a. ,BHD“: Durchmesser $=8,9, \ldots, 127[\mathrm{~cm}]$

b. „ANZAHL“: Anzahl Bäume pro Durchmesser,

c. "VOLUMEN“: Durchschnittsvolumen pro Durchmesser $\left[\mathrm{dm}^{3}\right]$,

d. "TARIF“: Tarifvolumen pro Durchmesser [ $\left.\mathrm{dm}^{3}\right]$,

e. eine grafische Darstellung von VOLUMEN/TARIF. Damit kann leicht überblickt werden, ob und wie stark TARIF größer, gleich oder kleiner als VOLUMEN für jeden Durchmesser ist, d. h. wie sich der Tarif den Daten anpaßt,

f. dem mittleren Baumvolumen für den Tarif und seiner Standardabweichung.

4. Informative Meldungen und Fehlermeldungen:

a. Fehler in den Leitkarten,

b. grobe Fehler in den Inventurdaten,

c. Überschreitung der Anzahl erlaubter Gruppen oder Tarife.

d. Ein Entwicklungstarif oder gar ein Gesamttarif kann nicht berechnet werden, weil zu wenig Bäume vorhanden sind oder die Spanne der Durchmesser zu klein ist,

e. in einer Gruppe kommt nur ein Tarif vor (War das beabsichtigt?),

f. es werden keine Daten auf die Inventurdatei 2 zugewiesen, weil eine Entwicklungsstufe ohne berechenbaren Tarif ist.

Die Inventurdatei 2 enthält alle Daten der Inventurdatei 1 und zusätzlich das Volumen gemäß zugehörigem Tarif. Die Inventurdatei 2 wird in den Auswerte- und Prognoseprogrammen weiterverwendet.

Die Tarifkarten enthalten die Funktionswerte

$$
T(D)\left[\mathrm{dm}^{3}\right] \text { für } \mathrm{D}=8,9, \ldots, 120[\mathrm{~cm}]
$$

für alle Tarife parallel in einem übersichtlichen Format. 
Tabelle 1

Tarifliste $\mathrm{BHD}=$ Brusthöhendurchmesser; ANZAHL = Anzahl Meßbäume; VOLUMEN = Durchschnittsvolumen pro Meßbaum; TARIF = Wert der Tariffunktion; VOLUMEN/TARIF: Für VOLUMEN > TARIF steht das Zeichen „1“ rechts der mittleren Punktkolonne, für = darauf und für < links davon, so z.B. für BHD $=8,127,11 \mathrm{~cm}$

List of a tarif BHD = diameter at $1.3 \mathrm{~m}$; ANZAHL $=$ number of sample trees; VOLUMEN $=$ mean volume per sample tree; TARIF = value of the tarif function; VOLUMEN/TARIF: for VOLUMEN $>$ TARIF the character , 1 “ lies to the right of the central column of dots, for $=$ on it, and for $<$ to the left of it, e.g. for BHD $=8,127,11 \mathrm{~cm}$

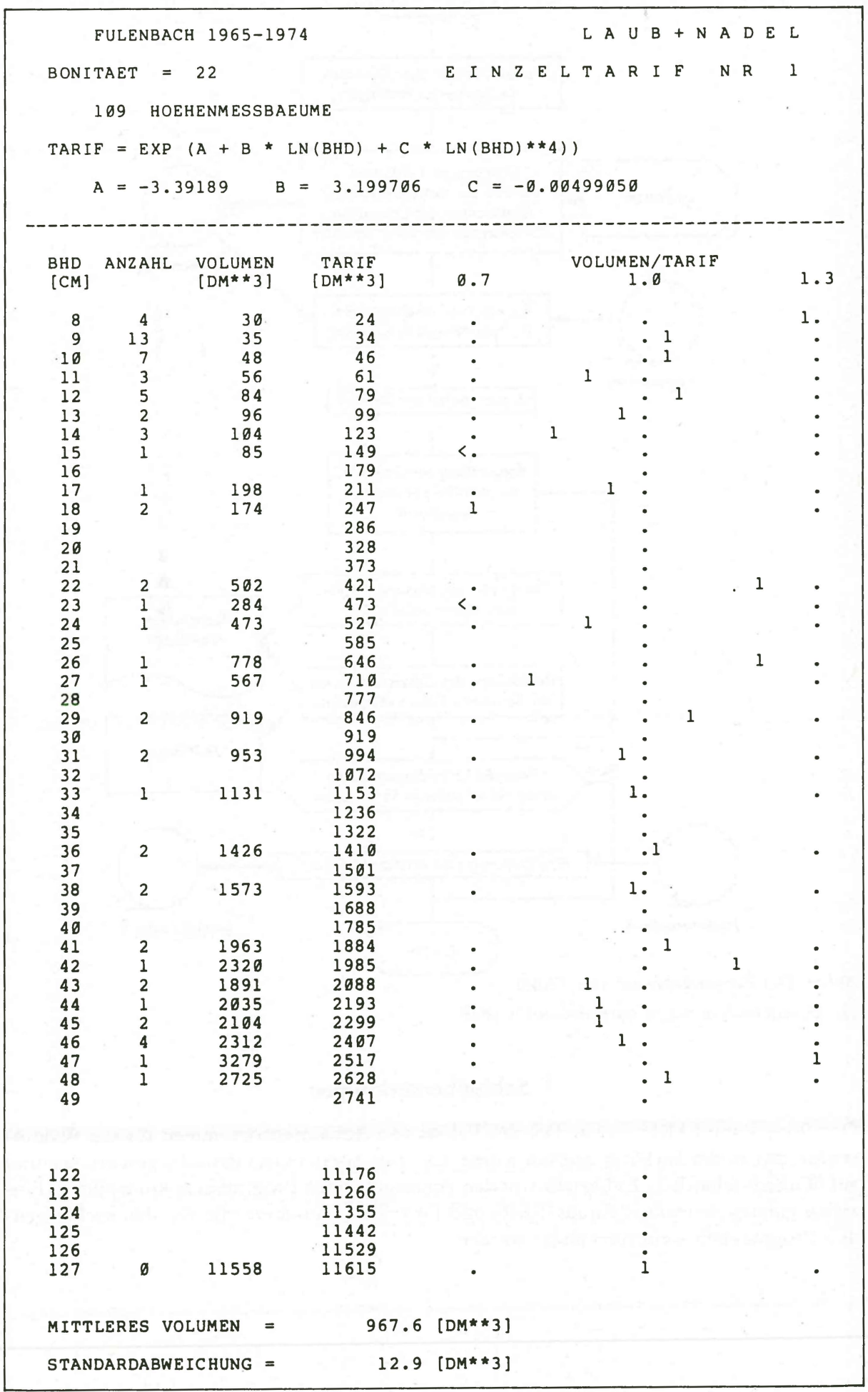




\subsection{Der Programmablauf}

In Abbildung 6 ist der Ablauf des Programms in seinen wesentlichen Zügen angegeben.

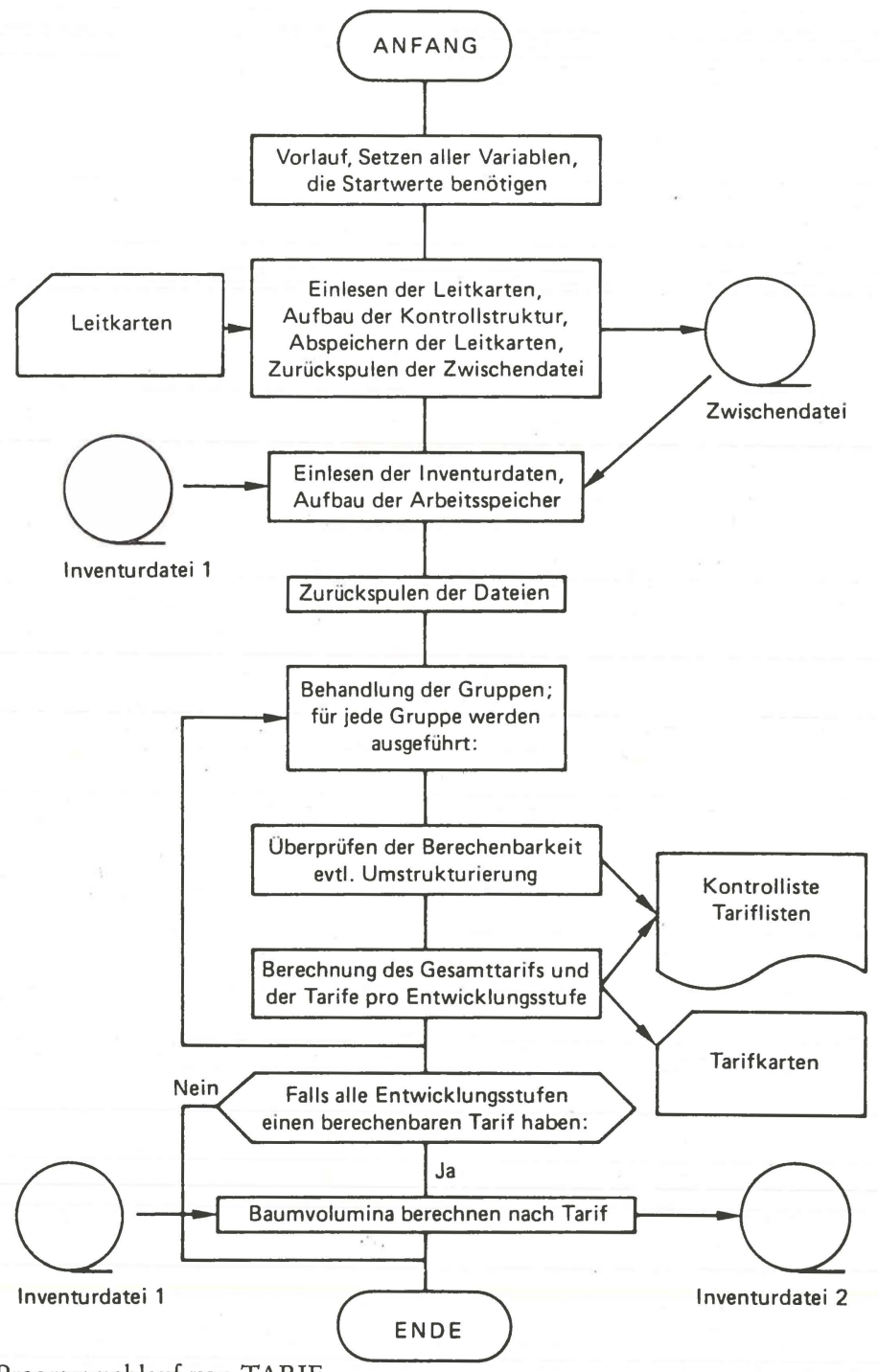

Abb. 6. Der Programmablauf von TARIF

Fig. 6. Sequence of major operations of TARIF

\section{Schlußbemerkungen}

Das Programm TARIF ist ein Teil des Pakets von Auswerteprogrammen für die Waldinventur, das an der EAFV entwickelt wurde. Es ist in ANSI-FORTRAN IV geschrieben und auf Wunsch erhältlich. Es bezieht von den vorausgehenden Programmen kontrollierte Einzelbaumdaten, berechnet daraus Tarife und Einzelbaumvolumina, die von den nachfolgenden Programmen weiterverwendet werden. 
Über Leitkarten können verschiedene Varianten angesteuert werden: verschiedene Aufteilungen in Tarife für Entwicklungsstufen, getrennte oder gemeinsame Tarife für Laubholz und Nadelholz.

Die gewählte Form der Funktion

$$
T(D)=e^{a_{1}+a_{2} \ln D+a_{3} \ln 4 D}
$$

ist sehr gut geeignet als Tariffunktion. Sie besitzt genügend Flexibilität, sich den Daten anzupassen, andererseits ist sie steif genug, die Schwankungen der gemessenen Volumenwerte auszugleichen. Sie wurde seit über einem Jahrzehnt mit Erfolg im Vorgängerprogramm SAMPLE3 verwendet.

Die Tariffunktionen, so wie sie vom Programm TARIF geliefert werden, passen meist gut bis sehr gut auf die Daten. Die Reduktion der Zahl der freien Parameter auf zwei durch eine separat abgeleitete Parameterregression bringt Vorteile und Nachteile. Sie versteift die Tariffunktionen und bewirkt, daß die Tarife für aufsteigende Entwicklungsstufen (Jungwuchs, Stangenholz, dünnes, dickes Baumholz) etwas konsequenter wachsende Volumina für gleiche Durchmesser ausweisen. Dafür zeigen die Tarife eine leichte Tendenz, für $\mathrm{D}<15 \mathrm{~cm}$ systematisch zu große oder zu kleine Volumina zu liefern, wohingegen die Tarife mit drei freien Parametern dort bessere Resultate liefern.

Das Programm benötigt auf dem CDC-6400/6500-Rechner der ETH Zürich etwa 17000 (60-bit-) Worte Speicherplatz, dazu 6000 für die Systemunterstützung. Es läuft ebenfalls auf IBM 370 und UNIVAC 1100.

Die Verarbeitungskosten für einen typischen Tarif mit 1000 BHD-Meßbäumen betragen etwa Fr. 13,-. Davon entfallen $4 \%$ auf die Tarifberechnung, der Rest auf Ein- und Ausgabe der Daten.

Ich möchte den Herren P. Schmid-HaAs und E. Vogel danken für ihr Interesse an dieser Arbeit und die regen Diskussionen, die zum Gelingen wesentlich beigetragen haben.

\section{Zusammenfassung}

Tariffunktionen dienen dazu, allein aus dem Brusthöhendurchmesser das Volumen eines Baumes zu schätzen. Die zu diesem Zweck sehr geeigneten Funktionen $T(D)=\exp \left(a_{1}+\right.$ $a_{2} \ln D+a_{3} \ln 4$ D) werden vorgestellt. Ihre Parameter lassen sich anschaulich deuten. Trotz geringer Baumzahlen können Tarife für Entwicklungsstufen berechnet werden. Die Rolle der Gewichtung der Daten wird erörtert. Sodann werden die nichtlinearen KleinsteQuadrate-Gleichungen zur Bestimmung der Parameter hergeleitet. Zu ihrer Lösung müssen durch Linearisierung geeignete Startwerte berechnet werden, die mit dem Newtonverfahren dann weiter verbessert werden. Das Computerprogramm erlaubt die Berücksichtigung der Aufnahmewahrscheinlichkeit, verschiedene Kombinationen von Waldteilen zu Entwicklungsstufen und die Berechnung von sowohl getrennten als auch gemeinsamen Tarifen für Laubholz und Nadelholz.

\section{Summary \\ The computation of tarifs for forest inventories}

Tarif functions are used to estimate the volume of a tree only from its diameter at breast height. Functions of the form $T(d b h)=\exp \left(a_{1}+a_{2} \ln d b h+a_{3} l^{4} d b h\right)$ are very well suited for that purpose. Their parameters are easy to interpret. In spite of small numbers of sample trees tarifs can be computed for development stages. The role of weighting is discussed. In a longer chapter the non-linear least squares equations for the determination of the parameters are derived. In order to solve them, starting values have to be obtained from a suitable linearisation of the problem. In a Newton algorithm the exact solutions are then 
approximated. The computer program allows for varying sampling probabilities, various combinations of stands according to stages of development, and for the computation of separate as well as combined tarifs for broadleaved trees and conifers.

\section{Literatur}

Cochran, W. G., 1972: Stichprobenverfahren. Berlin/New York: de Gruyter. S. 113.

Hoffmann, C., 1982a: Das Programm TARIF: Programmbeschreibung. Eidg. Anst. forstl. Versuchswesen.

Hoffmann, C., 1982b: Nonlinear Curve Fitting for Prediction (in Vorbereitung).

Mahrer, F., 1981: Mündliche Mitteilung.

PARDE, J., 1961: Dendrométrie. Gap: Louis-Jean, S. 147

Schmid-HAAs, P., 1968: Anleitung für das Tarifprogramm SAMPLE 3, Eidg. Anst. forstl. Versuchswesen.

Schmid-HaAs, P.; Roiko-Jokela, P.; Mingard, P.; Zobeiry, M., 1971: The optimal determination of the volume of standing trees. Mitt. forstl. Bundesvers. anst. Wien, 91, S. 33-54.

Schmid-HaAs, P.; Werner, J.; Baumann, E., 1978: Kontrollstichproben: Aufnahmeinstruktion. 2. Aufl. Eidg. Anst. forstl. Versuchswesen, Ber. 186, $57 \mathrm{~S}$.

VoGEL, E., 1981: Kontrollstichproben: Auswerteprogramme, Benützerhandbuch. Eidg. Anst. forstl. Versuchswesen.

Anschrift des Verfassers: Dr. C. Hoffmann, Eidg. Anstalt für das forstliche Versuchswesen, $\mathrm{CH}-8903$ Birmensdorf 